\title{
Dynamic Optimal Random Access for Vehicle-to-Roadside Communications
}

\author{
Man Hon Cheung*, Fen Hou ${ }^{\dagger}$, Vincent W.S. Wong*, and Jianwei Huang ${ }^{\dagger}$ \\ ${ }^{*}$ Department of Electrical and Computer Engineering, \\ The University of British Columbia, Vancouver, Canada \\ ${ }^{\dagger}$ Department of Information Engineering, \\ The Chinese University of Hong Kong, Hong Kong, China \\ Emails: * ${ }^{\{}$mhcheung, vincentw $\} @$ ece.ubc.ca, ${ }^{\dagger}\{$ fhou, jwhuang $\} @$ ie.cuhk.edu.hk
}

\begin{abstract}
In a drive-thru scenario where vehicles drive by a roadside access point (AP) to obtain temporary Internet access, it is important to design efficient resource allocation schemes to fully utilize the limited communication opportunities. In this paper, we study the random access problem in drivethru communications in a dynamic environment, where both the channel contention level and channel capacity vary over time. We assume that a vehicle has a file to upload when it is within the coverage range of the AP. The vehicle will pay a fixed amount each time it tries to access the AP, and will incur a penalty if it cannot finish the file uploading when leaving the AP. We first formulate the optimal transmission problem as a finite-horizon sequential decision problem. Then we solve the problem using dynamic programming, and design a dynamic optimal random access algorithm. Simulation results based on a realistic vehicular traffic model show that our algorithm achieves the minimal total cost, the highest probability of completing file upload, and the highest upload ratio as compared with two other heuristic schemes.
\end{abstract}

\section{INTRODUCTION}

The development of intelligent transportation system (ITS) has gained significant momentum in recent years, especially after the Federal Communications Commission (FCC) in the United States allocated $75 \mathrm{MHz}$ licensed spectrum in the 5.9 $\mathrm{GHz}$ band in 1999 for this purpose. There are two types of application in an ITS. Safety applications, such as cooperative forward collision warning, lane change warning, and left turn assistant (e.g., [1], [2]), have been proposed to improve the safety of the passengers by informing the vehicles of potential dangers ahead of time. Non-safety applications, such as traffic management, instant messaging, and media content delivery, have been designed to avoid traffic congestion and improve the experience of driving.

Vehicular ad hoc networks (VANETs), which are designed to provide reliable communications among vehicles and roadside access points (APs), are playing an important role in the development of ITS. VANETs support the ITS applications through different types of communication patterns, including vehicle-to-roadside (V2R) and vehicle-to-vehicle (V2V) communications [3]. V2R communications involve data transmissions between vehicular nodes and roadside APs, and V2V communications involve data exchange among vehicular nodes only. For both types, we can further classify the communications as either single-hop or multi-hop. In this paper, we focus on single-hop V2R uplink transmission from the vehicles to the $A P$. Due to the limited communication opportunities between the AP and the vehicles, efficient resource allocation in V2R communications is crucial for the successful deployment of ITS applications.

A number of previous results have been reported on the resource allocation in V2R communications. Zhang et al. in [4] considered the case where roadside APs only store the data uploaded by the vehicles. Scheduling priority is determined by two factors: data size and deadline. A request with a smaller data size or an earlier deadline will be served first. Hadaller et al. in [5] proposed a scheduling protocol that grants channel access to a vehicle with the maximum transmission rate. Analytical and simulation results showed significant overall system throughput improvement over a benchmark scheme. Jarupan et al. in [6] proposed a cross-layer protocol for V2R multi-hop communication. The medium access control (MAC) module collects information of local data traffic, and the routing module finds a route with the minimum delay. Niyato et al. in [7] considered the coalition formation of vehicles to improve bandwidth utilization. Coalitions based on either maximizing the individual utility or the aggregate utility were studied. Tan et al. in [8] analyzed the performance of a downlink resource allocation scheme in a V2R communication system with one AP on a road. The distribution of the number of bytes downloaded per drive-thru was derived using Markov reward processes.

Most of these results only proposed heuristic resource allocation schemes in VANETs. This motivates us to design an optimal uplink resource allocation scheme analytically. Moreover, we aim to design a random access algorithm that is distributed in nature, so that it is compatible with the IEEE 802.11p standard which is developed to facilitate the provision of wireless access in vehicular networks [9].

In this work, we consider the drive-thru scenario [10], where vehicles pass by an AP located along the road, and obtain Internet access for only a limited amount of time. We assume that a vehicle wants to upload a file to the AP when it is within the coverage range of the AP. The AP charges the vehicle in proportion to the number of time slots that it requests for packet transmission. It also provides the vehicle with information on the current contention level within the 


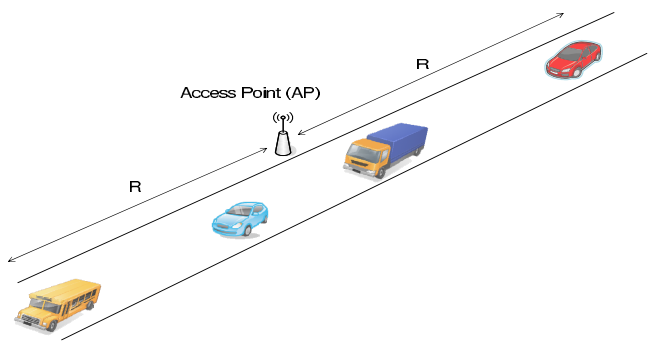

Fig. 1. Drive-thru vehicle-to-roadside (V2R) communications.

coverage range. The vehicle needs to decide when to transmit by taking into account the required payment, the probability of completing file upload, and the level of contention within the coverage range.

The main contributions of our work are as follows:

- Problem formulation: We formulate the optimal transmission problem as a finite-horizon sequential decision problem.

- Algorithm design: We apply dynamic programming to design an optimal random access algorithm for this dynamic scenario.

- Performance evaluation: Simulation results show that our proposed algorithm achieves the minimal total cost, the highest probability of completing file upload, and the highest upload ratio as compared with two other heuristic schemes.

The rest of the paper is organized as follows. We describe our system model in Section II, and formulate our problem as a finite-horizon sequential decision problem in Section III. Our dynamic programming based algorithm is proposed in Section IV. Simulation results are given in Section V, and the paper is concluded in Section VI.

\section{SySTEM MODEL}

We consider a drive-thru scenario as shown in Fig. 1, where an AP is installed on a highway to provide Internet services to vehicles within its coverage range. We assume that the AP has a transmission radius $R$. We focus on the uplink transmission from a vehicle to the AP, where the vehicle wants to upload a file of size $S$ when it moves through the coverage range of the AP.

\section{A. Traffic Model}

Let $\lambda$ be the average number of vehicles passing by the fixed AP per unit time. We assume that the number of vehicles moving into the coverage range of the AP follows a Poisson process with mean arrival rate $\lambda$. Let $\rho$ be the vehicle density representing the number of vehicles per unit distance along the road segment, and $\nu$ be the speed of the vehicles. From [11], we have

$$
\lambda=\rho \nu .
$$

The relation between the vehicle density $\rho$ and speed $\nu$ is given by the following equation [11]:

$$
\nu=\nu_{f}\left(1-\rho / \rho_{\max }\right),
$$

where $\nu_{f}$ is the free-flow speed when the vehicle is moving on the road without any other vehicles, and $\rho_{\max }$ is the vehicle density during traffic jam.

As we are studying the traffic flow in the steady state, all the vehicles within the coverage range are assumed to move with the same speed $\nu$ in (2). The sojourn time of each vehicle in the coverage range of the AP is given by

$$
T_{\text {soj }}=2 R / \nu \text {. }
$$

The maximum number of vehicles that can be accommodated in the segment of the coverage range is given by

$$
N_{\max }=\left\lfloor 2 R \rho_{\max }\right\rfloor,
$$

where $\lfloor\cdot\rfloor$ denotes the floor function.

\section{B. Channel Model}

Wireless signal propagations suffer from path loss, shadowing, and fading. Since the distance between the vehicle and the AP varies over time in the drive-thru scenario, the dominant effect of channel attenuation is the path loss attenuation. The channel capacity at time slot $t$ is given by

$$
w_{t}=W \log _{2}\left(1+\frac{P}{N_{0} W d_{t}^{\gamma}}\right),
$$

where $W$ is the channel bandwidth, $P$ is the transmit power of the vehicle, $d_{t}$ is the distance between the AP and vehicle at time slot $t$, and $\gamma$ is the path loss exponent. We assume that the additive white Gaussian noise has a zero mean and a power spectral density $N_{0} / 2$.

\section{System Operations}

We consider a slotted MAC protocol where time is divided into equal time slots of length $\Delta t$. We assume that there is perfect synchronization between the AP and the vehicles with the use of global positioning system (GPS). The total number of time slots that the vehicle stays within the coverage range of the AP is $T=\left\lfloor\frac{2 R}{\nu \Delta t}\right\rfloor$. We define the set of time slots as $\mathcal{T}=\{0, \ldots, T-1\}$.

When the vehicle first enters the coverage range of the AP, it declares to the AP the type of application that it supports. In return, the AP provides the vehicle with information related to the channel contention in the system ( $\lambda$ and $\left.p^{s u c c}\right)$, channel capacity in all the time slots $\left(w_{t}, \forall t \in \mathcal{T}\right)$, the price $(q)$, and the estimated number of vehicle departures from the coverage range in all the time slots $\left(l_{t}, \forall t \in \mathcal{T}\right)$. We further elaborate these system parameters as follows:

- $p^{\text {succ }}$ represents the probability that a vehicle can successfully gain assess to the current time slot for data transmission when all the vehicles in the coverage range contend for this time slot. $p^{s u c c}$ is estimated by the AP to indicate the level of system contention and it varies over time. Since the level of system contention is related to the number of vehicles $n$ in the coverage range, we define $p^{s u c c}=f(n)$, where $f$ is a strictly decreasing function of $n$. The AP knows the value of $n$, since vehicles need 
to establish and terminate their connections when they enter and leave the coverage range, respectively.

- $q \geq 0$ denotes the payment of a vehicle to the AP for each time slot that it sends a transmission request.

- $l_{t}$ represents the number of vehicle departures at time slot $t$. Since the vehicles move with constant speed $\nu$ in the traffic model, we assume that $\left(l_{t}, \forall t \in \mathcal{T}\right)$ are accurately known by the AP, and are sent to the vehicle when it enters the coverage range.

In each time slot, the AP first broadcasts the value of $p^{s u c c}$. If a vehicle decides to transmit within this time slot, it then sends a request to the $\mathrm{AP}$ at its scheduled minislot, where $N_{\max }$ mini-slots are reserved for transmission requests. The transmissions of requests are thus collision-free. After collecting the requests from the vehicles in the coverage range, the AP assigns this time slot to one of these vehicles based on the $p^{s u c c}$ announced. The vehicle, which receives the acknowledgement (ACK), can transmit the data packets in the remaining time $\Delta t_{d a t a}$ of this time slot, where $\Delta t_{\text {data }}<\Delta t$.

Meanwhile, regardless of which vehicle is granted the time slot, each vehicle sending a request in the time slot will pay $q$ to the AP. Without such pricing, the vehicle would send requests in every time slots, which prevents the efficient allocation of time slots to the most needed application.

The vehicle aims to achieve a good tradeoff between file upload completion and the total payment to the AP: If it waits until it is close to the AP and the channel is better, it may finish uploading the file within a small number of time slots and thus with a small amount of payment. However, if it starts to transmit too late, then there may not be enough time to finish the file upload before leaving the coverage range. This is true especially when $p^{\text {succ }}$ is low, the file size $S$ is large, and the total number of time slots $T$ is small (e.g., due to high vehicle speed $\nu$ ). Therefore, it is very challenging for vehicles to decide when to request for data transmission.

\section{PROBLEM Formulation}

In this section, we formulate the problem of finding the optimal transmission policy of the vehicle as a finite-horizon sequential decision problem [12]. The decision epochs are

$$
t \in \mathcal{T}=\{0, \ldots, T-1\} .
$$

The state of the system is defined as $\left(s, p^{s u c c}\right)$, where the state element $s \in \mathcal{S}=[0, S]$ represents the remaining size (in bits) of the file to be uploaded. For $t \in \mathcal{T}$, the number of vehicles in the coverage range of the $\mathrm{AP}$ is $n \in \mathcal{N}=$ $\left\{1, \ldots, N_{\max }\right\}$. So $\mathcal{P}=\{f(n): n \in \mathcal{N}\}$.

At any state $\left(s, p^{s u c c}\right)$, the vehicle has two possible actions:

$$
a \in \mathcal{A}=\{0,1\},
$$

where action $a=1$ implies that the vehicle decides to request to transmit, and action $a=0$ otherwise. Notice that $\mathcal{A}$ is the set of possible actions and is independent of time.

The cost at state $\left(s, p^{s u c c}\right)$ with chosen action $a$ at time slot $t$ is

$$
c_{t}\left(s, p^{s u c c}, a\right)=a q, \quad \forall t \in \mathcal{T} \text {. }
$$

After the vehicle has left the coverage range at time slot $T$, we define the self-incurred penalty of the vehicle for not being able to finish the file uploading as

$$
\hat{c}_{T}\left(s, p^{s u c c}\right)=h(s)
$$

where $h(s) \geq 0$ is a nondecreasing function of $s$ with $h(0)=$ 0 . The function is chosen depending on the quality of service (QoS) requirement of the application.

The state transition probability $p_{t}\left(\left(s^{\prime}, p^{s u c c^{\prime}}\right) \mid\left(s, p^{s u c c}\right), a\right)$ is the probability that the system will go into state $\left(s^{\prime}, p^{s u c c^{\prime}}\right)$ if action $a$ is taken at state $\left(s, p^{s u c c}\right)$ at time slot $t$. Since the transition from $p^{s u c c}$ to $p^{s u c c^{\prime}}$ is independent of the value of $s$, we have

$$
\begin{aligned}
& p_{t}\left(\left(s^{\prime}, p^{s u c c^{\prime}}\right) \mid\left(s, p^{s u c c}\right), a\right) \\
= & p_{t}\left(s^{\prime} \mid\left(s, p^{s u c c}\right), a\right) p_{t}\left(p^{s u c c^{\prime}} \mid p^{s u c c}\right) .
\end{aligned}
$$

With action $a=1$, we have

$$
p_{t}\left(s^{\prime} \mid\left(s, p^{s u c c}\right), 1\right)= \begin{cases}p^{s u c c}, & \text { if } s^{\prime}=\left[s-w_{t} \Delta t_{d a t a}\right]^{+} \\ 1-p^{s u c c}, & \text { if } s^{\prime}=s \\ 0, & \text { otherwise }\end{cases}
$$

where $[x]^{+}=\max \{0, x\}$. The first and second cases correspond to the scenarios of successful and unsuccessful packet transmissions, respectively. With action $a=0$, we have

$$
p_{t}\left(s^{\prime} \mid\left(s, p^{s u c c}\right), 0\right)= \begin{cases}1, & \text { if } s^{\prime}=s \\ 0, & \text { otherwise }\end{cases}
$$

where the size of the remaining file to upload does not change. For the transition probability of $p^{s u c c}$, we have

$$
\begin{aligned}
& p_{t}\left(p^{s u c c^{\prime}} \mid p^{s u c c}\right)=p_{t}\left(f\left(n^{\prime}\right) \mid f(n)\right)=p_{t}\left(n^{\prime} \mid n\right) \\
& = \begin{cases}\frac{(\lambda \Delta t)^{n^{\prime}-n+l_{t+1}}}{\left(n^{\prime}-n+l_{t+1}\right) ! \phi(n, t)}, & \text { if } n-l_{t+1} \leq n^{\prime} \leq N_{\max }, \\
0, & \text { otherwise, }\end{cases}
\end{aligned}
$$

where $\phi(n, t)=\sum_{y=0}^{N_{\max }-n+l_{t+1}} \frac{(\lambda \Delta t)^{y}}{y !}$ is a normalization factor. As shown in the first line of (13), because $p^{\text {succ }}=f(n)$ is a strictly decreasing function of $n$, there is a one-to-one mapping between $p^{\text {succ }}$ and $n$. The second line describes the probability with $n^{\prime}-n+l_{t+1}$ arrivals due to the Poisson process and $l_{t+1}$ deterministic departures at time $t+1$. $n^{\prime}$ is lower-bounded by $n-l_{t+1} \geq 0$ when there is no vehicle arrival, and is upper-bounded by $N_{\max }$.

Let $\delta_{t}: \mathcal{S} \times \mathcal{P} \rightarrow \mathcal{A}$ be a function that specifies the transmission decision of the vehicle at state $\left(s, p^{\text {succ }}\right)$ at time slot $t$. We define a policy as $\pi=\left(\delta_{t}\left(s, p^{\text {succ }}\right), \forall s \in\right.$ $\left.\mathcal{S}, p^{\text {succ }} \in \mathcal{P}, t \in \mathcal{T}\right)$. We denote $\left(s_{t}^{\boldsymbol{\pi}}, p_{t}^{\text {succ, } \boldsymbol{\pi}}\right)$ as the state at time slot $t$ if policy $\pi$ is used, and we let $\Pi$ be the feasible set of $\boldsymbol{\pi}$. The vehicle aims to find an optimal policy $\boldsymbol{\pi}^{*}$ that minimizes the total expected cost when it is within the coverage range of the AP, which can be formulated as the 
following optimization problem

$$
\begin{gathered}
\min _{\boldsymbol{\pi} \in \Pi} E_{\boldsymbol{\pi},\left(S, p_{0}^{s u c c}\right)}\left[\sum_{t=0}^{T-1} c_{t}\left(s_{t}^{\boldsymbol{\pi}}, p_{t}^{\text {succ }, \boldsymbol{\pi}}, \delta_{t}\left(s_{t}^{\boldsymbol{\pi}}, p_{t}^{\text {succ }, \boldsymbol{\pi}}\right)\right)\right. \\
\left.+\hat{c}_{T}\left(s_{T}^{\boldsymbol{\pi}}, p_{T}^{\text {succ }, \boldsymbol{\pi}}\right)\right],
\end{gathered}
$$

where $E_{\boldsymbol{\pi},\left(S, p_{0}^{\text {succ }}\right)}$ denotes the expectation with respect to the probability distribution by policy $\pi$ with an initial state $\left(S, p_{0}^{s u c c}\right)$.

\section{Finite-Horizon DyNAMIC PROGRAMMING}

In this section, we solve problem (14) using the finitehorizon dynamic programming. This leads to a dynamic optimal random access algorithm that results in the minimal total cost in problem (14).

Let $v_{t}\left(s, p^{s u c c}\right)$ be the minimal expected total cost that the vehicle has to pay from time slot $t$ to $T$, given that the system is in state $\left(s, p^{s u c c}\right)$ immediately before the decision at time slot $t$. The optimality equation [12, pp. 83] relating the minimal expected total cost at different states for $t \in \mathcal{T}$ is

$$
v_{t}\left(s, p^{s u c c}\right)=\min _{a \in \mathcal{A}}\left\{\psi_{t}\left(s, p^{s u c c}, a\right)\right\}
$$

where

$$
\begin{aligned}
& \psi_{t}\left(s, p^{s u c c}, a\right)=c_{t}\left(s, p^{s u c c}, a\right)+ \\
& \sum_{s^{\prime} \in \mathcal{S}} \sum_{p^{s u c c^{\prime}} \in \mathcal{P}} p_{t}\left(\left(s^{\prime}, p^{s u c c^{\prime}}\right) \mid\left(s, p^{s u c c}\right), a\right) v_{t+1}\left(s^{\prime}, p^{s u c c^{\prime}}\right) .
\end{aligned}
$$

The first and second terms on the right hand side of (16) are the immediate cost and the expected future cost in the remaining time slots for choosing action $a$, respectively. For $t=T$, we have the boundary condition that

$$
v_{T}\left(s, p^{s u c c}\right)=\hat{c}_{T}\left(s, p^{s u c c}\right)=h(s) .
$$

The value of $\psi_{t}\left(s, p^{s u c c}, 0\right)$ and $\psi_{t}\left(s, p^{s u c c}, 1\right), \forall t \in \mathcal{T}$, can be obtained as follows:

Lemma 1:

$$
\begin{aligned}
\psi_{t}\left(s, p^{s u c c}, 0\right)= & \sum_{\substack{m=0 \\
N_{\max }-n+l_{t+1}}} \frac{(\lambda \Delta t)^{m}}{m ! \phi(n, t)} \\
& \times v_{t+1}\left(s, f\left(n+m-l_{t+1}\right)\right),
\end{aligned}
$$

and

$$
\begin{gathered}
\psi_{t}\left(s, p^{s u c c}, 1\right)=q+\sum_{m=0}^{N_{\max }-n+l_{t+1}}\left[\frac{(\lambda \Delta t)^{m}}{m ! \phi(n, t)}\right. \\
\times\left[p^{s u c c} v_{t+1}\left(\left[s-w_{t} \Delta t_{d a t a}\right]^{+}, f\left(n+m-l_{t+1}\right)\right)\right. \\
\left.\left.+\left(1-p^{s u c c}\right) v_{t+1}\left(s, f\left(n+m-l_{t+1}\right)\right)\right]\right]
\end{gathered}
$$

where $n=f^{-1}\left(p^{s u c c}\right)$.

Proof: The results follow directly by evaluating $\psi_{t}\left(s, p^{s u c c}, a\right)$ in (16) using (10)-(13) for the cases $a=0$ and $a=1$.

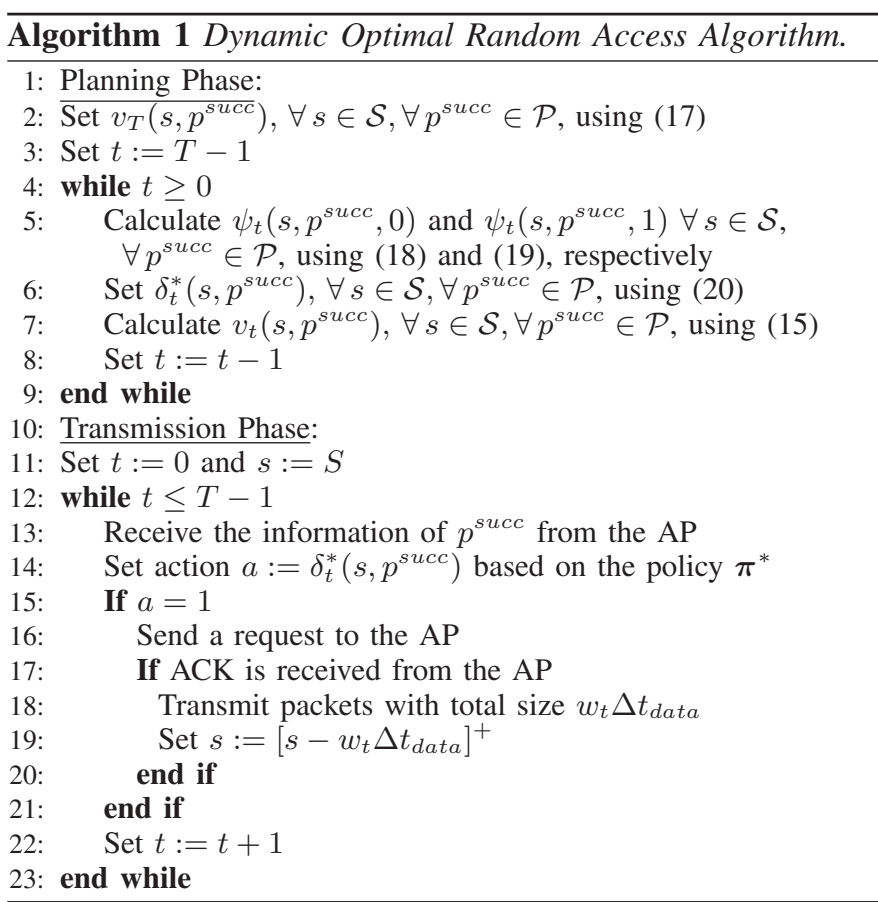

Using the optimality equation and backward induction [12, pp. 92], we are ready to obtain the optimal policy $\pi^{*}$. First, we obtain $v_{t}\left(s, p^{s u c c}\right), \forall s \in \mathcal{S}, p^{s u c c} \in \mathcal{P}$, by updating it recursively from time slot $t=T-1$ to time slot $t=0$ using (15)-(17). The optimal policy is described in Theorem 1, and is obtained as in the planning phase in Algorithm 1.

Theorem 1: We define the policy $\pi^{*}=\left(\delta_{t}^{*}\left(s, p^{s u c c}\right), \forall s \in\right.$ $\mathcal{S}, p^{\text {succ }} \in \mathcal{P}, t \in \mathcal{T}$ ) where

$$
\delta_{t}^{*}\left(s, p^{s u c c}\right)= \begin{cases}1, & \text { if } \psi_{t}\left(s, p^{s u c c}, 0\right)>\psi_{t}\left(s, p^{s u c c}, 1\right), \\ 0, & \text { otherwise }\end{cases}
$$

which is updated recursively from $t=T-1$ to $t=0$. It is the optimal solution of problem (14).

Proof: The decision rule in (20) corresponds to the decision in the optimality equation in (15). Using the principle of optimality [13, pp. 18], we can show that $\pi^{*}$ is the optimal solution of problem (14).

As a result, $\pi^{*}$ is a contingency plan that contains information about the optimal decision at all the states $\left(s, p^{s u c c}\right)$. We then propose our dynamic optimal random access algorithm in Algorithm 1. In the planning phase, the optimal policy $\pi^{*}$ is computed offline using dynamic programming. In the transmission phase, transmission decisions are made according to the optimal policy $\pi^{*}$, and $s$ is updated depending on whether the time slot is granted to the vehicle for transmission or not.

\section{PERformance Evaluations}

In this section, we evaluate the performance of Algorithm 1 by comparing it with two heuristic schemes using the traffic model described in Section II-A. The first scheme is a greedy 
TABLE I

SIMULATION PARAMETERS

\begin{tabular}{|c|c|}
\hline Parameters & Values \\
\hline AP's transmission radius $R$ & $100 \mathrm{~m}$ \\
\hline Free-flow speed $\nu_{f}$ & $110 \mathrm{~km} / \mathrm{hr}$ \\
\hline Vehicle jam density $\rho_{\max }$ & $100 \mathrm{veh} / \mathrm{km}$ \\
\hline Duration of a time slot $\Delta t$ & $0.02 \mathrm{sec}$ \\
\hline Duration for data transmission in a time slot $\Delta t_{\text {data }}$ & $0.018 \mathrm{sec}$ \\
\hline Bandwidth $W$ & $20 \mathrm{MHz}$ \\
\hline Transmit signal-to-noise ratio $\frac{P}{N_{0} W}$ & $60 \mathrm{~dB}$ \\
\hline Path loss exponent $\gamma$ & 3 \\
\hline Payment per time slot $q$ & 0.005 \\
\hline Self-incurred penalty $h(s)=K, \forall s>0$ & 100 \\
\hline Contention window $c w \in\left[c w_{\min }, c w_{\max }\right]$ & {$[1,8]$} \\
\hline
\end{tabular}

algorithm, in which each vehicle sends transmission requests at all the time slots if its file upload is not complete. That is, the greedy algorithm aims to maximize the probability of completing file upload. The second scheme is the exponential backoff algorithm that is similar to the one used in the IEEE 802.11. We have slightly modified it for the system that we consider as follows. Each vehicle has a counter, which randomly and uniformly chooses an initial integer value cnt from the interval $[0, c w)$, where $c w$ is the contention window size. The value of cnt decreases by one after every time slot. When $\mathrm{cnt}=0$, the vehicle will send a request. If the vehicle has sent a request in a time slot, the size of $c w \in\left[c w_{\min }, c w_{\max }\right]$ changes according to the response from the AP. If an ACK is received from the AP, $c w=c w_{\text {min }}$. Otherwise, $c w$ is doubled until it reaches $c w_{\max }$.

For performance evaluation, we run each experiment in 1000 different network scenarios and take the average value. We assume that the vehicle under consideration sends the transmission requests based on the given scheme. The requests are sent using the allocated mini-slots. We assume that the AP allows the vehicles to share the channel with equal probability. Therefore, $p^{\text {succ }}=f(n)=1 / n$ equals to the probability that the vehicle can successfully obtain a time slot. We consider a safety application with a strict delay requirement, where the whole file must be uploaded when the vehicle is within the coverage range since the partially uploaded data is useless. We set the self-incurred penalty as

$$
h(s)= \begin{cases}K, & \text { if } s>0, \\ 0, & \text { if } s=0,\end{cases}
$$

where $K$ is a positive constant. By setting $K>>q$, we place a higher importance on completing the file upload than on minimizing the total payment. The three schemes are evaluated using a similar transmission phase as in Algorithm 1, but with $\delta_{t}^{*}\left(s, p^{s u c c}\right)$ in line 14 replaced by the corresponding policies. The simulation parameters are listed in Table I.

First, we plot the total cost and the probability of completing file upload against the file size $S$ with $\nu=100 \mathrm{~km} / \mathrm{hr}$ in Figures. 2 and 3, respectively. It is clear from Fig. 2 that

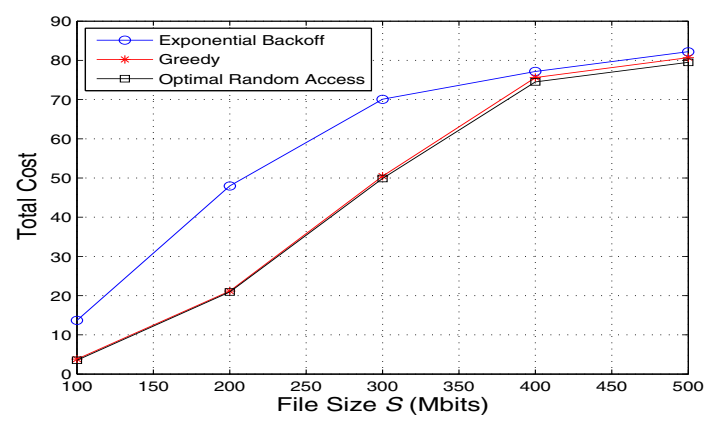

Fig. 2. Total cost versus file size $S$ for $\nu=100 \mathrm{~km} / \mathrm{hr}$. The optimal random access scheme has the minimal total cost (i.e., the total payment to the AP plus the self-incurred penalty).

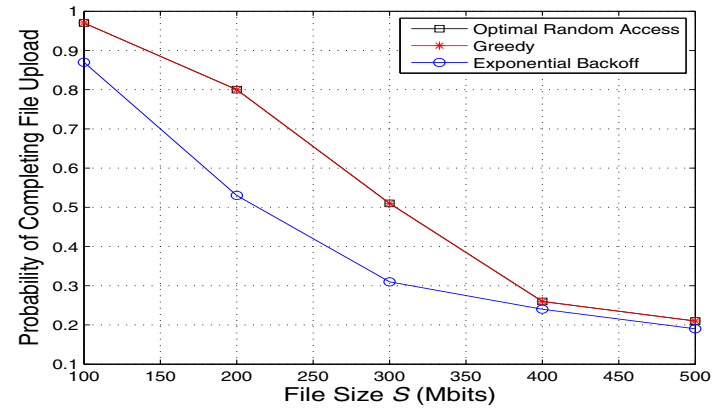

Fig. 3. Probability of completing file upload versus file size $S$ for $\nu=100$ $\mathrm{km} / \mathrm{hr}$. Notice that the greedy scheme aims to achieve the highest probability of completing file upload by design. The optimal random access scheme achieves the same optimal performance as this benchmark scheme.

our proposed scheme achieves the minimal total cost as stated in Theorem 1. In Fig. 3, we take the greedy scheme as the benchmark. We can observe that the optimal random access achieves the same probability of completing file upload as this benchmark scheme.

To measure the cost effectiveness of the file upload completion for the three schemes, we propose a metric called the upload ratio, which is equal to the probability of completing file upload divided by the total payment to the AP. In other words, it represents the chance of completing file upload that a vehicle can achieve per unit payment. Since optimal random access has the highest probability of completing file upload, while maintaining a small payment to the AP, it achieves the highest upload ratio as shown in Fig. 4.

Next, we study the impacts of traffic density $\rho$ on the total cost, the probability of completing file upload, and the upload ratio against $\rho$ for the file size $S=200$ Mbits in Figures. 5, 6, and 7 , respectively. We can see that the optimal random access scheme has the minimal total cost, the highest probability of completing file upload, and the highest upload ratio across different $\rho$. It is interesting to note that the total cost is small and the probability of completing file upload is high at both the very low and very high traffic densities cases. First, for a low $\rho$, the channel contention is small and the chance of completing the file upload is relatively high, which results in 


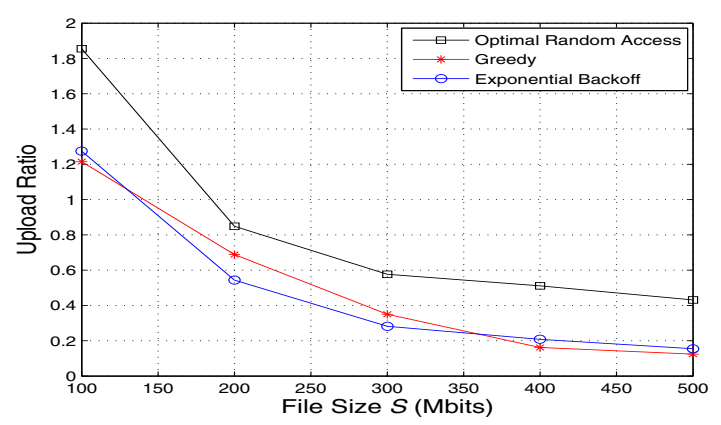

Fig. 4. Upload ratio (i.e., probability of completing file upload / total payment to the AP) versus file size $S$ for $\nu=100 \mathrm{~km} / \mathrm{hr}$. The optimal random access scheme achieves the highest upload ratio.

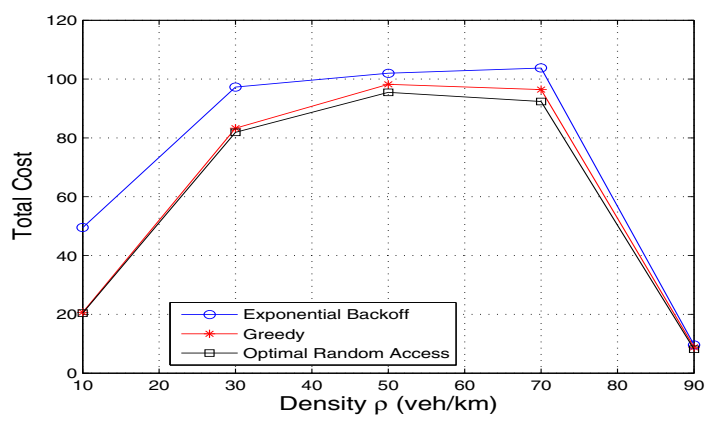

Fig. 5. Total cost versus traffic density $\rho$ for file size $S=200$ Mbits.

a small total cost. On the other hand, for a high $\rho$, the speed of the vehicle $\nu$ is low due to (2), which results in a large sojourn time $T_{\text {so } j}$ due to (3). As a result, the vehicle has more time to upload the file, which contributes to the high probability of completing file upload and thus a small total cost. However, the upload ratio is low due to the large payment required when the channel contention is heavy.

\section{CONCLUSION}

In this paper, we studied V2R uplink transmission in a dynamic drive-thru scenario, where both the channel contention level and channel capacity vary over time. We proposed an optimal random access algorithm based on the analysis of finite-horizon dynamic programming. Simulation results show that our scheme achieves the minimal total cost, the highest probability of completing file upload, and the highest upload ratio comparing with two other heuristic schemes. For future work, we will consider the setting with multiple APs, where coordination among APs is required for the file upload.

\section{ACKNOWLEDGMENT}

This research is funded by AUTO21, a member of the Network of Centres of Excellence of Canada program. It is also supported by the General Research Funds (Project Number 412509) established under the University Grant Committee of the Hong Kong Special Administrative Region, China, and the Global Scholarship for Research Excellence of the Chinese University of Hong Kong.

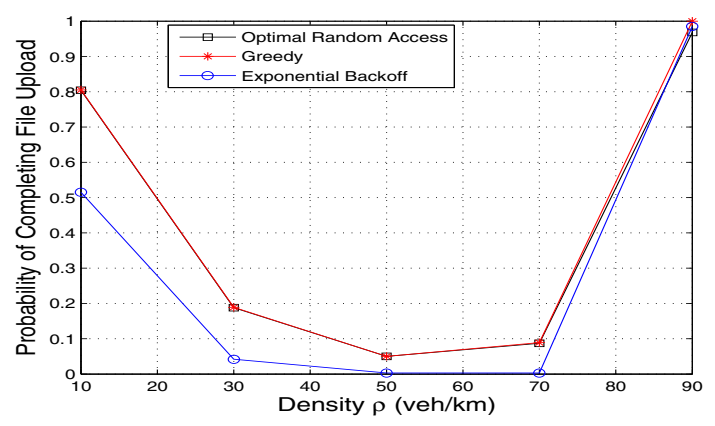

Fig. 6. Probability of completing file upload versus traffic density $\rho$ for file size $S=200$ Mbits

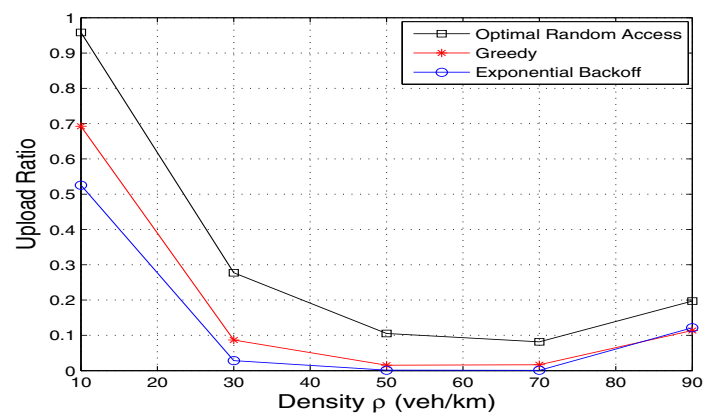

Fig. 7. Upload ratio versus traffic density $\rho$ for file size $S=200$ Mbits.

\section{REFERENCES}

[1] H. Hartenstein and K. P. Laberteaux, "A tutorial survey on vehicular ad hoc networks," IEEE Communications Magazine, vol. 46, no. 6, pp. 164-171, June 2008.

[2] Y. Toor, P. Muhlethaler, A. Laouiti, and A. De La Fortelle, "Vehicle ad hoc networks: Applications and related technical issues," IEEE Communications Surveys \& Tutorials, vol. 10, no. 3, pp. 74-88, 2008.

[3] E. Hossain, G. Chow, V. C. M. Leung, R. D. McLeod, J. Misic, V. W. S. Wong, and O. Yang, "Vehicular telematics over heterogeneous wireless networks: A survey," Elsevier Computer Communications, vol. 33, no. 7, pp. 775-793, May 2010.

[4] Y. Zhang, J. Zhao, and G. Cao, "On scheduling vehicle-roadside data access," in Proc. of ACM International Workshop on VANETs, Montreal, Quebec, Canada, Sept. 2007.

[5] D. Hadaller, S. Keshav, and T. Brecht, "MV-MAX: Improving wireless infrastructure access for multi-vehicular communication," in Proc. of ACM SIGCOMM Workshop, Pisa, Italy, Sept. 2006.

[6] B. Jarupan and E. Ekici, "Location- and delay-aware cross-layer communication in V2I multihop vehicular networks," IEEE Communications Magazine, vol. 47, no. 11, pp. 112-118, Nov. 2009.

[7] D. Niyato, P. Wang, W. Saad, and A. Hjorungnes, "Coalition formation games for bandwidth sharing in vehicle-to-roadside communications," in Proc. of IEEE WCNC, Sydney, Australia, Apr. 2010.

[8] W. L. Tan, W. C. Lau, and O. Yue, "Modeling resource sharing for a road-side access point supporting drive-thru Internet," in Proc. of ACM International Workshop on VANETs, Beijing, China, Sept. 2009.

[9] Wireless LAN Medium Access Control (MAC) and Physical Layer (PHY) Specification, IEEE Std. 802.11p, 2010.

[10] J. Ott and D. Kutscher, "Drive-thru Internet: IEEE 802.11b for automobile users," in Proc. of IEEE INFOCOM, Hong Kong, China, Mar. 2004.

[11] J. D. Fricker and R. K. Whitford, Fundamentals of Transportation Engineering: A Multimodal Systems Approach. Prentice Hall, 2004.

[12] M. L. Puterman, Markov Decision Processes: Discrete Stochastic Dynamic Programming. New York, NY: John Wiley and Sons, 2005.

[13] D. P. Bertsekas, Dynamic Programming and Optimal Control: Volume 1, 2nd ed. Athena Scientific, 2000 